\title{
Frequency response of an atomic force microscope in liquids and air: Magnetic versus acoustic excitation
}

\author{
Elena T. Herruzo and Ricardo Garcia ${ }^{\text {a) }}$ \\ Instituto de Microelectronica de Madrid, CSIC, Isaac Newton 8, Tres Cantos, 28760 Madrid, Spain
}

(Received 22 May 2007; accepted 15 September 2007; published online 2 October 2007)

\begin{abstract}
We discuss the dynamics of an amplitude modulation atomic force microscope in different environments such as water and air. Experiments, analytical expressions, and numerical simulations show that the resonance curves depend on the excitation method used to drive the cantilever, either mechanical or magnetic. This dependence is magnified for small force constants and quality factors, i.e., below $1 \mathrm{~N} / \mathrm{m}$ and 10, respectively. We show that the equation for the observable, the cantilever deflection, depends on the excitation method. Under mechanical excitation, the deflection involves the base and tip displacements, while in magnetic excitation, the cantilever deflection and tip displacement coincide. (c) 2007 American Institute of Physics. [DOI: 10.1063/1.2794426]
\end{abstract}

Amplitude modulation (tapping mode) atomic force microscopy (AM-AFM) is nowadays the most flexible and versatile scanning probe microscope technique. ${ }^{1-4}$ In amplitude modulation AFM, a sharp probe is excited at a fixed frequency, usually near or at the first flexural resonance frequency while the probe is scanned in a raster fashion across the sample surface. The probe-surface interaction forces lead to a reduction of the amplitude of the oscillation from its free value. In the imaging mode, the amplitude reduction is used as a feedback parameter to map the topography of the surface. Two different cantilever driving methods are currently in use: mechanical also know as acoustic $^{5-7}$ and magnetic. ${ }^{8-10}$

In the acoustic excitation, a piezoactuator in contact with the microcantilever chip holder drives its oscillation. In the magnetic excitation, it is an oscillating magnetic field beneath the sample which drives the cantilever oscillation. Magnetic excitation requires cantilevers coated with magnetic particles; for that reason, most of the experiments in environments with relatively high $Q$ factors (air) are performed with the mechanical excitation method. The use of acoustic excitation in liquids is also common although it usually introduces unwanted mechanical resonances in the frequency response. ${ }^{11,12}$ On the other hand, a magnetic driving force generates canonical resonance curves both in air and liquids. ${ }^{10,13}$ It has been widely assumed that both excitation methods could be described with the same equations, although Lantz et al. used the proper expression for the oscillation amplitude in the acoustic excitation. ${ }^{13}$ The assumption that both methods could be described by the same equation was reinforced by the fact that theoretical and numerical simulations of AM-AFM in air provided a good qualitative and quantitative comparison with the experiments. ${ }^{14}$

Recently, several experiments and simulations have pointed out the existence of remarkable differences in the dynamic behavior of AFM microcantilevers in liquids depending on the excitation mode. ${ }^{15-19}$ Experiments performed by Volkov et al. ${ }^{16}$ showed that the resonance curve of a fluiddriven cantilever tends at high frequencies to a finite value of the oscillation amplitude. This is at variance with the observed behavior of magnetically excited cantilevers that

\footnotetext{
${ }^{\text {a) }}$ Author to whom correspondence should be addressed. Electronic mail:
} rgarcia@imm.cnm.csic.es show resonance curves with negligible oscillation amplitudes at high frequencies ${ }^{10,13}$ [see also Figs. 1(a) and 1(b)]. Legleiter et $a l .{ }^{17}$ and Jan et $a l .{ }^{18}$ have also studied some theoretical aspects of this problem.

In this letter, we investigate the influence of the excitation method on the resonance behavior of AFM driven cantilevers. To simplify the study, we just focus on the analysis of the frequency response of the first flexural resonance. We show that although the dynamic response of a cantilever depends on the excitation method, the observed differences (mechanical versus magnetic) depend on both the cantilever force constant and quality factor $Q$. For high force constants and $Q$ values, the differences are negligible while they are
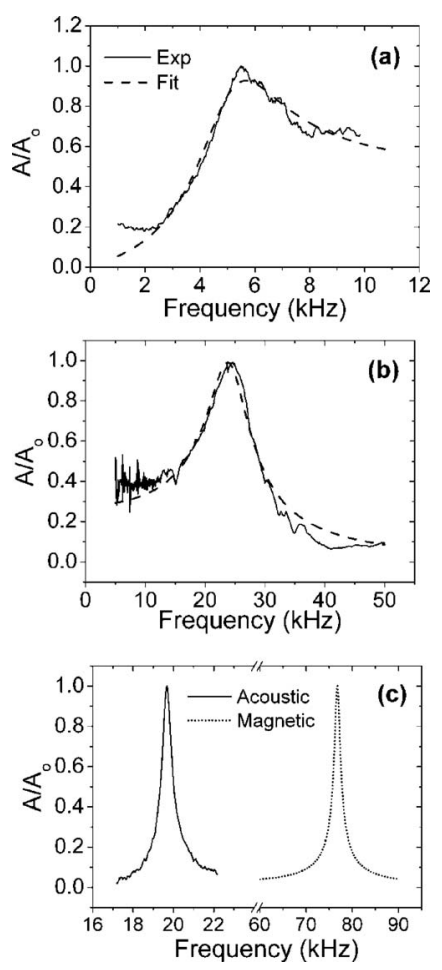

FIG. 1. Experimental resonance curves in water and air of mechanical and magnetically excited microcantilevers. (a) Dependence of the amplitude vs the frequency in water acoustic driven cantilevers. (b) For magnetic driven cantilevers. The dashed lines are the fittings given by Eqs. (5) and (8). (c) Frequency response in air for magnetic (circles) and acoustic driven cantilevers. Two different cantilevers have been used. 


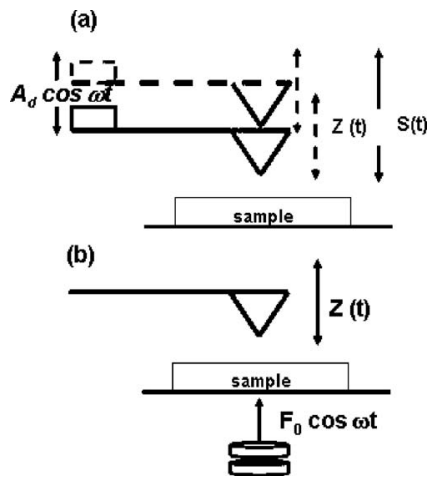

FIG. 2. Schematic of the relevant coordinates in mechanical and magnetically excitation methods. (a) Mechanical (acoustic) excitation and (b) magnetically excitation.

magnified at low force constants and quality factors. The observed differences can be traced back to the equation of motion that follows the cantilever deflection. Under mechanical excitation, the deflection involves both the base and tip displacements, while in magnetic excitation, the cantilever deflection and tip displacement are identical.

The acoustic excitation experiments in water were performed with an open fluid cell ${ }^{12}$ to avoid the spurious resonances associated with closed fluid cells. Magnetic excitation curves were obtained with a homemade excitation unit that was associated with a commercial AFM head (Pico SPM from Molecular Imaging, Tempe, AZ) and electronics (Nanotec Electronics, Madrid, Spain). Mechanical excitation was performed with a commercial instrument (Nanoscope IV from Veeco, Santa Barbara, CA). The force constant and quality factor (liquid) of the commercial cantilevers (BioLever, Olympus, Tokyo, Japan) were $(0.75 \mathrm{~N} / \mathrm{m}, 3)$ and $(0.2 \mathrm{~N} / \mathrm{m}, 1.5)$, respectively. The quality factors have been determined by fitting the experimental curves with the proper analytical expressions (see below). The back side of the cantilevers was coated by using triode sputtering in ultrahigh vacuum (base pressure of $10^{-9}$ mbar) with a film of $5 \mathrm{~nm} \mathrm{Ti}$ and $60 \mathrm{~nm}$ Co. The simulations were obtained by solving numerically the equation of motion with a fourth RungeKutta algorithm.

Presently, several theoretical approaches are used to describe the amplitude modulation AFM operation, such as semianalytical methods, ${ }^{20,21}$ three-dimensional finite element fluid-structure models, ${ }^{22}$ one-dimensional continuous models, ${ }^{23,24}$ or point-mass models. ${ }^{25-28}$ Despite its apparent simplicity, point-mass models render a qualitative and semiquantitative comparison with experiments. ${ }^{14,17,18}$

The deflection of an AFM microcantilever driven by a magnetic force in fluids and far from the sample surface, can be described by ${ }^{28}$

$$
\ddot{z}(t)+\frac{b}{m^{*}} \dot{z}(t)+\frac{k}{m^{*}} z(t)=\frac{F_{0}}{m^{*}} \cos \omega t,
$$

where $z(t)=z_{0}+A \cos \omega t$ is the tip-cantilever's deflection [Fig. 2(a)], $\omega$ is the excitation angular frequency in liquid, $F_{0}$ is the driving force, $k$ is the force constant of the cantilever, $m^{*}$ is the effective cantilever mass in liquid, and $b$ is the coefficient of the damping force. The effective mass includes the cantilever mass in air and the fluid mass in contact with the cantilever (added mass). It is calculated by ${ }^{29,30}$ the cantilever (added mass). It is calculated by ${ }^{29,30}$ tion $(k=0.75 \mathrm{~N} / \mathrm{m}$ and $Q=3)$ show the expected behavior of
Downloaded 18 Jun 2009 to 161.111 .235 .43 . Redistribution subject to AlP license or copyright; see http://apl.aip.org/apl/copyright.jsp

$$
m^{*}=0.24 m+0.144 \rho(w L)^{3 / 2}=\frac{k}{\omega_{0}^{2}} .
$$

The density of the fluid is $\rho$, and the coefficient $b$ is calculated by using the string of spheres model, ${ }^{31}$

$$
b=\frac{L}{w}\left(\frac{3}{4} \pi w^{2} \sqrt{2 \eta \rho \omega}+3 \pi w \eta\right),
$$

where $L$ is the cantilever length, $w$ is the cantilever width, $m$ is the cantilever mass, and $\eta$ is the fluid viscosity; $\omega_{0}$ is the resonance angular frequency if damping is absent. Then, the effective quality factor

$$
Q=\frac{m^{*} \omega_{0}}{b} \text {. }
$$

The amplitude of the oscillation for Eq. (1) is the well-know Lorentzian expression of

$$
A(\omega)=\frac{\left(F_{0} / k\right) \omega_{0}^{2}}{\sqrt{\left(\omega_{0}^{2}-\omega^{2}\right)^{2}+\left(\frac{\omega \omega_{0}}{Q}\right)^{2}}} .
$$

For small excitation frequencies, $A$ tends to $F_{0} / k$, while for frequencies well above $\omega_{0}$ the amplitude vanishes.

On the other hand, the motion of an AFM tip driven by a piezoactuator can be described by

$$
\ddot{S}(t)+\frac{b}{m^{*}} \dot{S}(t)+\omega_{0}^{2} z(t)=0,
$$

where $z(t)$ and $S(t)$ are the cantilever deflection and the tip displacement, respectively, as described in Fig. 2(a). Because the optical beam deflection method measures directly the cantilever slope, the above equation has to be rewritten in terms of the observable (deflection),

$$
z(t)=S(t)-A_{d} \cos \omega t=z_{0}+A_{z} \cos \omega t,
$$

where $A_{d}$ is the amplitude of the base displacement. Then, the amplitude is given by ${ }^{13}$

$$
A_{z}(\omega)=\frac{F_{0} / k \sqrt{\omega^{4}+\left(\frac{\omega \omega_{0}}{Q}\right)^{2}}}{\sqrt{\left(\omega_{0}^{2}-\omega^{2}\right)^{2}+\left(\frac{\omega \omega_{0}}{Q}\right)^{2}}} .
$$

Expression (8) shows that for $\omega \rightarrow 0$, the amplitude goes to zero $\left(A_{z} \rightarrow 0\right)$. In the other limit, for $\omega \rightarrow \infty$, the amplitude goes to $A_{z}=F_{0} / k$. The above behavior is in contrast with the result found previously [Eq. (5)] that gave $A=F_{0} / k(\omega \rightarrow 0)$ and $A=0(\omega \rightarrow \infty)$. At resonance $\left(\omega=\omega_{0}\right)$ Eq. (8) gives

$$
A_{z}=\frac{Q F_{0}}{k}\left(1+\frac{1}{Q^{2}}\right)^{1 / 2}
$$

while for the magnetic excitation [Eq. (5)], we obtain the well-known result of $A=Q F_{0} / k$.

The experimental curves shown in Fig. 1 reveal the differences between the frequency response of magnetic and acoustic cantilevers. The frequency response for a cantilever excited acoustically $(k=0.2 \mathrm{~N} / \mathrm{m}$ and $Q=1.5)$ shows that the amplitude is rather small at low frequencies, while it seems to converge at high frequencies [Fig. 1(a)]. On the other hand, the resonance curves obtained under magnetic excita- 

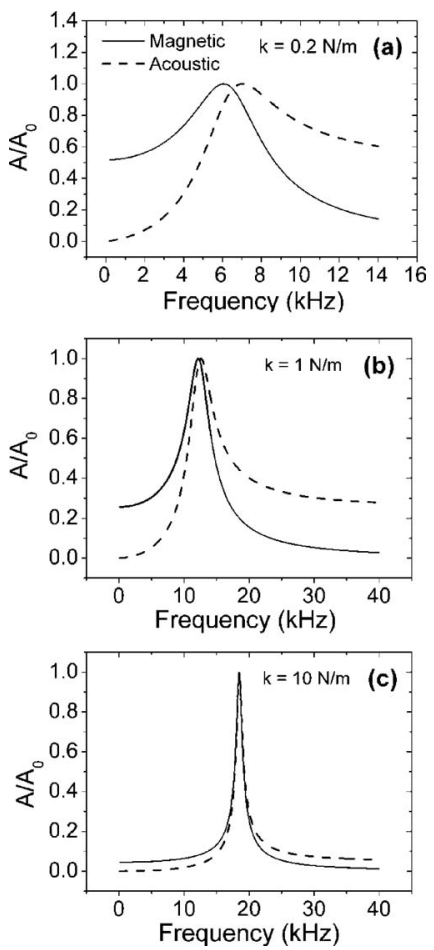

FIG. 3. Theoretical acoustic and magnetic resonance curves for different cantilevers in water (a) $k=0.2 \mathrm{~N} / \mathrm{m}$, (b) $k=1 \mathrm{~N} / \mathrm{m}$, and (c) $k=10 \mathrm{~N} / \mathrm{m}$. The curves have been obtained for $Q=2$.

a Lorentzian curve [Fig. 1(b)]; i.e., the amplitude goes to a finite value for low frequencies, reaches the maximum at the resonance, and then goes to zero at high frequencies. The dashed lines are the fittings obtained by using Eq. (5) (magnetic) and Eq. (8) (acoustic). The above differences tend to disappear when the same cantilevers are driven in air [Fig. $1(\mathrm{c})]$.

The theoretical curves shown in Fig. 3 describe the behavior of magnetic and acoustic excited cantilevers with low quality factor $(Q=2)$. Magnetic excitation shows Lorentzianlike resonance curves, while acoustic excitation shows a curve that has zero amplitude values at low frequencies and tends to saturate for frequencies higher than the resonance. The differences are marked for low force constant cantilevers, let say $k<1 \mathrm{~N} / \mathrm{m}$. The resonance peak happens at higher frequencies for acoustically driven cantilevers, again the differences decrease by increasing the cantilever force constant above $1 \mathrm{~N} / \mathrm{m}$ [Figs. 3(a)-3(c)].

The theoretical simulations also show that the frequency response is nearly independent of the excitation method for cantilevers with high quality factors $Q \geqslant 50$. The above findings can be explained by comparing the equations that describe the amplitude for magnetic excitation [Eq. (5)] and acoustic excitation [Eq. (8)]. We find a very good agreement between the analytical expressions [Eqs. (5) and (8)] and the numerical simulations of Eqs. (1) and (6), respectively.

In summary, we have described the dynamic behavior of an amplitude modulation AFM microcantilever far from the sample surface. Our description is valid for both low and high quality factor environments. It shows that the excitation method either acoustic or magnetic does influence the resonance curves. Magnetic excitation provides canonical resonance curves, while acoustic excitation provides resonance curves that tend to zero amplitude at low frequencies and saturate at high frequencies (water). This behavior is magnified by using cantilevers with small force constants. For high quality factors (air), the resonance curves are similar. We explain the observed behavior and relate it to the fact that the equation of motion for the cantilever deflection depends on the excitation method.

While this paper was under submission, two related articles were published. ${ }^{32,33}$

This work was financially supported by the European Commission (FORCETOOL) and the Ministerio de Educación y Ciencia (MAT2006-03833). We do thank stimulating discussions with J.R. Lozano, S. Patil and N.F. Martinez.

${ }^{1}$ R. Garcia, R. Magerle, and R. Perez, Nat. Mater. 6, 405 (2007).

${ }^{2}$ T. Ando, N. Kodera, E. Takai, D. Maruyama, K. Saito, and A. Toda, Proc. Natl. Acad. Sci. U.S.A. 98, 12468 (2001).

${ }^{3}$ C. Stroh, A. Ebner, M. Geretschlärger, G. Freudenthaler, F. Kienberger, A. S. M. Kamruzzahan, S. J. Smith-Gill, H. J. Gruber, and P. Hinterdorfer, Biophys. J. 87, 1981 (2004).

${ }^{4}$ M. Tello et al., Adv. Mater. (Weinheim, Ger.) 17, 1480 (2005).

${ }^{5}$ Q. Zhong, D. Inniss, K. Kjoller, and V. B. Elings, Surf. Sci. Lett. 290, L688 (1993).

${ }^{6}$ P. K. Hansma et al., Appl. Phys. Lett. 64, 1738 (1994).

${ }^{7}$ C. A. J. Putman et al., Appl. Phys. Lett. 64, 2454 (1994).

${ }^{8}$ E. L. Florin, M. Radmacher, B. Fleck, and H. E. Gaub, Rev. Sci. Instrum. 65, 639 (1993).

${ }^{9}$ S. J. O'Shea, M. E. Welland, and J. B. Pethica, Chem. Phys. Lett. 223, 336 (1994).

${ }^{10}$ W. Han, S. M. Lindsay, and T. Jing, Appl. Phys. Lett. 69, 4111 (1996).

${ }^{11}$ T. E. Schäffer, J. P. Cleveland, F. Ohnersorge, D. A. Walters, and P. K. Hansma, J. Appl. Phys. 80, 3622 (1996).

${ }^{12}$ A. Maali, C. Hurth, T. Cohen-Bouhacina, G. Couturie, and J. P. Aimé, Appl. Phys. Lett. 88, 163504 (2006).

${ }^{13}$ M. Lantz, Y. Z. Liu, X. D. Cui, H. Tokumoto, and S. M. Lindsay, Surf. Interface Anal. 27, 354 (1999).

${ }^{14}$ R. Garcia et al., Phys. Rev. Lett. 97, 016103 (2006).

${ }^{15}$ I. Revenko and R. Proksch, J. Appl. Phys. 87, 526 (2000).

${ }^{16}$ A. O. Volkov, J. S. Burnell-Gray, and P. K. Datta, Appl. Phys. Lett. 85, 5397 (2004).

${ }^{17}$ J. Legleiter, M. Park, B. Cusik, and T. Kowalewski, Proc. Natl. Acad. Sci. U.S.A. 103, 4813 (2006)

${ }^{18}$ C. Jan, T. Cohen-Bouhacina, and A. Maali, Appl. Phys. Lett. 90, 113512 (2007).

${ }^{19}$ F. J. Rubio-Sierra, R. Vazquez, and R. W. Stark, IEEE Trans. Nanotechnol. 5, 692 (2006).

${ }^{20}$ C. P. Green and J. E. Sader, J. Appl. Phys. 98, 114913 (2005).

${ }^{21}$ J. E. Sader, J. Appl. Phys. 84, 64 (1998).

${ }^{22}$ S. Basak, A. Raman, and S. V. Garimella, J. Appl. Phys. 99, 114906 (2006).

${ }^{23}$ T. R. Rodriguez and R. Garcia, Appl. Phys. Lett. 80, 1646 (2002).

${ }^{24}$ C. Rankl, V. Pastushenko, F. Kieberger, C. M. Stroh, and P. Hinterdorfer, Ultramicroscopy 100, 301 (2004).

${ }^{25}$ G. Y. Chen, R. J. Warmack, A. Hyuang, and T. Thundat, J. Appl. Phys. 78, 1465 (1995).

${ }^{26}$ A. San Paulo and R. Garcia, Phys. Rev. B 64, 193411 (2001).

${ }^{27}$ H. Hölscher and U. D. Schwarz, Appl. Phys. Lett. 89, 073117 (2006).

${ }^{28}$ J. Leigleiter and T. Kowalewski, Appl. Phys. Lett. 87, 163120 (2005).

${ }^{29}$ J. E. Greespon, J. Acoust. Soc. Am. 33, 1485 (1961).

${ }^{30}$ S. J. O'Shea and M. E. Welland, Langmuir 14, 4186 (1998).

${ }^{31}$ H. Hosaka, K. Itao, and S. Kuroda, Sens. Actuators, A 49, 87 (1995).

${ }^{32}$ J. Kokavecz and A. Mechler, Appl. Phys. Lett. 91, 023113 (2007).

${ }^{33}$ X. Xu and A. Raman, J. Appl. Phys. 102, 034303 (2007). 